\title{
La Première Guerre mondiale à la breughélienne : sur Breughel à l'Yser de Franz Hellens
}

\author{
The First World War à la breughélienne: \\ about Breughel à l'Yser by Franz Hellens
}

\author{
Julia Łukasiak \\ Université de Varsovie \\ e-mail: julialukasiak@student.uw.edu.pl
}

\begin{abstract}
The article shows the importance of a tale "Breughel à l'Yser" in the context of the Franz Hellens' identity transformation which takes place during the First World War. On the one hand, in his tale Hellens refers by different means to the Breughel's oeuvre continuing in that way the myth of Belgian writer perceived as a heir of the Flemish painters. On the other hand, via the landscape, the typical element of the Flemish master's style, the writer introduces the elements of fantasy into a realistic description of a battlefield, creating in that way his own aesthetics of "fantastique reel" (fantastic reality). Thus, this tale enables to examine how Hellens passes fluently from the "inherited identity" to the mature writer's identity.
\end{abstract}

Keywords: Hellens, Breughel, fantastic reality, First World War

Valérie Nahon perçoit les Documents secrets de Franz Hellens comme un texte dans lequel l'écrivain belge se construit soi-même en se définissant comme l'écrivain-peintre (Nahon, 2009). Il semble que ses écrits autobiographiques soient un exemple du fonctionnement de l'identité narrative. Le cas de Franz Hellens est un exemple d'une rare conformité de l'identité narrative individuelle et l'identité narrative collective. Rappelons que d'après David Carr l'identité narrative concerne aussi des communautés qui «ont une 'existence racontée', qu'elles existent dans la mesure où elles se constituent en unités narratives, comme sujets d'une histoire » (Carr, 1988: 212). De cette manière les nations fondent une identité en recourant 
à des mythes, des légendes et des événements historiques importants. Le problème de l'identité narrative collective semble être particulièrement important pour la génération de 1880. Tout au début de l'histoire des lettres belges, les écrivains tels que Camille Lemonnier, Maurice Maeterlinck ou Charles de Coster veulent se voir comme héritiers des peintres flamands. Nous retrouvons aussi l'écho de cette conception chez Franz Hellens selon lequel : "L'écrivain belge, le Flamand surtout, apporte dans sa façon de voir, de sentir et de s'exprimer, un reflet indéniable des tableaux des grands maîtres de la peinture flamande et wallonne : Roger de la Pasture, Jérôme Bosch, Breughel... Réaliste et mystique à la fois, il a un goût prononcé pour les couleurs vives et les formes corsées»(Hellens, 1990). La référence aux maîtres anciens permet, d'un côté, de gagner «la légitimité artistique » dans le milieu parisien où la peinture flamande est reconnue et, de l'autre, ce fantasme fait remonter l'histoire des lettres belges au $\mathrm{XVI}^{\mathrm{e}}$ siècle. Le mythe des écrivains-héritiers des peintres flamands est une des manifestations du concept picardien de l'âme belge inspiré par la théorie romantique de la race. Cependant, la Première Guerre mondiale met fin à ce mythe unitaire et provoque une crise identitaire. Comme le note Jean-Marie Klinkenberg, "l'instauration du suffrage universel [...] (1919) révèle un État partagé entre deux communautés linguistiques et culturelles distinctes. [...] Le mythe unitaire qui cimentait la Belgique vole dès lors en éclats » (Denis, Klinkenberg, 2005 : 147-148). En effet, les années de la guerre opèrent une certaine désillusion par rapport au champ littéraire belge, voire une crise identitaire que commentent deux historiens des lettres belges. Le premier, Marc Quaghebeur appelle les années après la guerre «le temps de doute » (Quaghebeur, 1998: 99) tandis que le second, Jean-Marie Klinkenberg, situe ces années à la frontière de la phase centrifuge et centripète ${ }^{1}$. Le cycle de « Six réalités de la guerre » de Franz Hellens, créé dans les années 1913-1916, s'inscrit dans l'époque de cette grande transition identitaire et l'écrivain, dans cette perspective, parait être un représentant typique de la génération 1880. Bien que l'identité de l'écrivain-peintre soit proche d'Hellens tout au long de sa carrière littéraire, les années de la guerre, comme le remarque Valérie Nahon, constituent pour lui un passage de "l'identité héritée » vers «l'identité reconvertie ». Dès 1913, en effet, l'écrivain se réoriente vers l'art français, ce qui est visible à la fois dans ses écrits d'art et dans ses romans ${ }^{2}$. Le conte "Breughel à l'Yser » révèle l'intérêt d'Hellens pour la peinture flamande : cette fois-ci Hellens non seulement se réfère

\footnotetext{
${ }^{1}$ Les phases centrifuge et centripète servent habituellement à périodiser l'histoire de la littérature belge francophone en rendant compte de son attitude face à la littérature française. Selon J.-M. Klinkenberg et Benoît Denis la phase centrifuge (éloignement du «centre» qui est Paris)) correspondrait à la période 1830-1920 et la phase centripète (rapprochement du «centre ») à la période 1920-1970/80. Voir à ce sujet B. Denis, J.-M. Klinkenberg, La littérature belge. Précis d'histoire sociale, Bruxelles, Labor, 2005.

${ }^{2}$ Valérie Nahon souligne surtout l'importance des contacts de l'écrivain belge avec Alexandre Archipenko et Henri Matisse dans la réorientation de l'écrivain vers l'art français (Nahon : 2009).
} 
à des tableaux breughéliens, mais il essaye de traduire, " du pictural au littéraire », des paysages breughéliens en les inscrivant dans le contexte de la Première Guerre mondiale. Le paysage chez Hellens est un outil du dépaysement, le facteur qui introduit un élément d'irréalité, de fantastique. Ainsi, l'analyse de ce conte permet de révéler comment Hellens utilise d'une manière créative l'héritage breughélien afin de créer sa propre théorie du fantastique réel.

Le cycle de six contes, "Six réalités de la guerre», constitue une version abrégée des écrits de la guerre d'Hellens. Pendant la guerre, l'écrivain partage le sort malheureux de la génération de 1880 : il n'est pas directement mobilisé ${ }^{3}$, mais pendant les premières années du conflit il vit «l'existence humiliante » d'un refugié, ce qui est la conséquence directe de l'invasion allemande. L'écrivain rentre au pays natal où il décide immédiatement « d'écrire [s] es impressions de la guerre » (Hellens, 1958 : 65). Ainsi Hellens part pour le front avec le pressentiment d'être témoin de son temps. Déguisé d'un uniforme d'aumônier militaire, il visite le front de Pervyse, d'Ypres et de Nieuport. Malheureusement, ses écrits de la guerre, comptant environ un millier de pages, intitulés Les Vainqueurs du Temps, ne trouvent aucun éditeur. En 1923, Hellens, cette fois-ci plus critique envers son ouvrage, décide de n'en publier que quelques chapitres, réunis sous le titre «Six réalités de la guerre», comme une annexe à la première édition des Réalités fantastiques (1923). Le changement du titre reflète un grand changement de la perspective historique. Le premier titre, Les Vainqueurs du Temps, annonce une description du point de vue des gagnants, tandis que le second, « Six réalités de la guerre », beaucoup moins pompeux, annonce une description des évènements vus «de l'intérieur ». Il semble que dans cette seconde approche Hellens rejoint le point de vue de Walter Benjamin qui, dans son texte inachevé « Sur le concept de l'histoire » (1940), s'oppose à la version de l'Histoire écrite par les vainqueurs en postulant que dans le travail d'historien «il s'agit pour le matérialisme historique de retenir l'image du passé qui s'offre inopinément au sujet historique à l'instant du danger » (Benjamin, 2000 : 431). En effet, dans « Six réalité de la guerre », Hellens présente quelques impressions du front de bataille, prises sur le vif, du point de vue d'un narrateur intradiégétique, qui n'est pas un héros de la guerre : il doute de sa « vocation » de soldat et a l'impression de s'y trouver un peu par hasard. Le titre du recueil dans lequel Hellens a publié ses écrits de la guerre, Réalités fantastiques, invite à voir ce texte non seulement comme une chronique subjective de l'époque, mais d'y chercher un côté imaginaire, fantastique. Cependant, dans les années trente, après avoir apporté quelques remarques stylistiques, Hellens décide d'exclure à jamais ces textes et cette étape de sa vie: ces contes ne réapparaîtront plus dans les éditions suivantes des Réalités fantastiques.

Comme le titre le laisse supposer, le conte « Breughel à l'Yser » contient maintes références à la peinture de Pieter Breughel l'Ancien. Dès les premières années de

\footnotetext{
${ }^{3}$ Hellens aurait pu être mobilisé, car il « avai[t] tiré un mauvais numéro », mais son père l'a fait remplacer par un jeune homme, comme c'était convenu dans sa classe sociale (Hellens, 1958: 65).
} 
son activité littéraire, Hellens montre un grand intérêt pour les arts plastiques, ce qui est typique pour les écrivains de la génération 1880. Il écrit des critiques d'art, peint lui-même et c'est justement l'échec dans ce domaine qui lui fait choisir la carrière littéraire. Dans les Documents secrets, il « [se] demande ce qui serait arrivé si [s]es peintures n'avaient pas été refusées » (Hellens, 1958 : 27) à une exposition. En effet, les premières années de sa carrière littéraire montrent la fascination de l'auteur pour la peinture des maîtres flamands et, en particulier, celle de Pieter Breughel l'Ancien. L'écrivain belge semble être particulièrement fasciné par deux toiles du peintre : Les Aveugles et le Massacre des Innocents. Les références aux Aveugles apparaissent déjà dans sa première œuvre En ville morte. Les scories (1906) et reviennent successivement dans Les hors-le-vent (1909) et dans les Clartés latentes (1912) ${ }^{4}$. La toile Le Massacre des Innocents constitue l'inspiration pour le drame Massacrons les Innocents (1911). Le conte « Breughel à l'Yser » clôt ce cycle en marquant ainsi la fin de la fascination hellensienne pour la peinture du maître flamand ${ }^{5}$.

Dans « Breughel à l'Yser », l'écrivain belge jongle avec plusieurs tableaux breughéliens, mais aucun d'eux n'y apparaît expressis verbis. Hellens joint les éléments de différentes toiles en dévoilant ainsi devant le lecteur son interprétation approfondie de la peinture breughélienne. Dans diverses biographies, le maître flamand est soit vu comme Pieter le Drôle (Van Mander, 1884), l'auteur de maintes kermesses telles que La Danse de la mariée en plein air (1566), Le Repas de noce (1568) ou La Danse des paysans (1568), soit comme le poète des gueux, chroniqueur du conflit cruel entre l'Espagne et les Pays-Bas (Baie, 1938). Hellens, dans son conte, relie les deux côtés de la peinture breughélienne, ludique et sérieux, en les mettant ensemble dans le même texte, ce qui est rare.

Selon Bob Claessens, l'expression « les fêtes breughéliennes » se référerait «à des banquets et des danses où l'on se gonfle de cochonnaille, se trémousse par couples et s'enivre sans vergogne » (Cleassens, 1969 : 1964). Dans le conte écrit par Hellens, au milieu de la guerre, le narrateur éprouve la nostalgie des kermesses d'autrefois : «J'ai faim [...] ; je ne frémirai pas de dégoût devant le rouge spectacle d'une kermesse de gosiers et de mâchoires, et j'y prendrai ma part comme au temps de mes ancêtres, paysans goulus et trapus, qui fêtaient leurs femmes et leurs récoltes à coups de gueule, jusqu'à en vomir » (Hellens, 1923 : 211). Cependant, tandis que les kermesses breughéliennes sont pleines de joie de vivre et annoncent une ambiance conviviale, la kermesse dépeinte par Hellens est cruelle. La frontière entre la kermesse et le massacre se révèle fragile et, dans le temps de la guerre, ils sont unis par la relation de cause-conséquence : «Il y aura grande fête tout à l'heure autour des chardons, $\underline{\text { car }}^{6}$ voici un peloton tout entier qui ramène un troupeau par les

\footnotetext{
${ }^{4}$ Dans ce recueil apparaît aussi la référence à une autre toile breughélienne, Les Chasseurs sur la neige.

${ }^{5}$ En 1962, Franz Hellens réédite Massacrons les Innocents, en y apportant quelques changements stylistiques sans grande importance pour le message du texte.

${ }^{6}$ C'est nous qui soulignons.
} 
cornes, pareil à des prisonniers criant pitié. Au-delà du ruisseau, des porcs s'étaient réfugiés; on les attaque à coups de fusil, et les hurlements de ces bêtes traquées sont si déchirants, qu'ils couvrent la voix du canon » (Hellens, 1923 : 214). Tout au long de ce passage le lecteur sent la terreur croissante du narrateur renforcée par la stylistique expressionniste. L'auteur anthropomorphise les animaux en les appelant les « prisonniers criant pitié » et en les comparant à des refugiés. Dans le contexte de la Première Guerre mondiale, nous pourrions percevoir ces animaux comme une représentation symbolique des victimes de la guerre. En créant cette métaphore, Hellens rejoint le point de vue de Breughel qui, en 1567, à une époque incertaine pour la Flandre, a créé deux versions du Massacre des Innocents. La version de Vienne représente la scène biblique mise dans un décor flamand du seizième siècle tandis que dans la version de Hampton Court, comme le remarque Bob Claessens, les Innocents sont remplacés par des animaux et des objets, « de telle sorte qu'il s'agit là moins du "Massacre des Innocents" que du "Pillage du village" » (Claessens, 1969 : 169). Dans chacun de ces tableaux, Breughel répète la composition comme un copiste, mais change l'objet de l'oppression de l'armée et, par ce procédé, il actualise la portée du thème biblique. La chasse aux animaux décrite dans le conte hellensien pourrait être lue come une allusion à un autre tableau du maitre flamand, Les Chasseurs sur la neige qui a déjà inspiré Hellens à écrire le conte sous le même titre (Clartés latentes, 1912). En comparant les deux textes, "Les chasseurs sur la neige » et « Breughel à l'Yser », inspirés par la même toile, il est possible de voir un changement dans la perception de la peinture breughélienne par l'écrivain belge. Dans le conte de 1912 a lieu une certaine sublimation de la chasse : les simples chasseurs sont tellement éblouis par la beauté d'un paon qu'ils le laissent voler. Dans «Breughel à l'Yser", par contre, le narrateur est le témoin d'une chasse cruelle et sanguinaire. C'est la guerre qui change son interprétation des tableaux du maitre flamand et la rend plus complexe. La lecture hellensienne permet de voir l'œuvre breughélienne comme une unité et de considérer deux faces de sa peinture en même temps : la face ludique et la face sérieuse. De cette manière ce conte apparaît comme une œuvre du connaisseur de la peinture breughélienne qui maîtrise les moindres détails de son art et sait les combiner habilement.

Franz Hellens ne s'arrête pas à une compilation créative de plusieurs tableaux breugheliens, mais il fait un pas de plus : il essaye de traduire la langue picturale du peintre. Pour Franz Hellens, Breughel est celui « qui peignit autrefois des hommes réels, trop réels, dans des paysages imaginaires» (Hellens, 1958: 41). Breughel est, en effet, un observateur perspicace de la campagne brabançonne, mais il semble qu'il peigne avec le même degré de réalisme les paysages et les paysans. Selon l'opinion du premier biographe du peintre, Karel van Mander, « on a pu dire de [Breughel] qu'en traversant les Alpes, il avait avalé les monts, et les rocs pour les vomir, à son retour, sur des toiles et des panneaux, tant il parvenait à rendre la nature avec fidélité » (Van Mander, 1805 : 299). Ainsi le caractère ima- 
ginaire des paysages breughéliens ne consiste pas en la mise des personnages dans un décor de l'au-delà, comme c'est souvent le cas des compositions de Jérôme Bosch. Comme le remarque Leo van Puyvelde, tandis que chez Jérôme Bosch, " c'est l'imagination créatrice qui prime[,] Breughel, lui, observe avec attention et intérêt, puis concrétise une image abstraite dans son esprit, tout en se souvenant de ses impressions » (Puyvelde, 1962: 157). Ainsi pour Breughel la source du fantastique se trouve dans la réalité. Nous retrouvons un écho lointain du procédé breughélien dans les écrits théoriques d'Hellens qui, une cinquantaine d'années plus tard, écrira : "Il existe une forme de fantastique qui touche de plus près à la réalité de tous les jours et du moment, et auquel on pourrait donner le nom du fantastique intérieur » (Hellens, 1967 : 105). Le fantastique issu de la réalité apparaît, semble-t-il, déjà dans "Six réalités de la guerre », ce que souligne Robert Frickx dans son commentaire de cette œuvre: «Son art consiste à nous faire basculer du concret dans l'imaginaire [...]. Cependant, cette réalité seconde ne nous est pas étrangère : rien de ce que le narrateur nous rapporte ne nous paraît vraiment inconnu » (Frickx, 1992: 90). Ainsi Hellens apparaît successeur du fantastique breughélien compris comme une certaine vision de la réalité qui dépayse le quotidien. Dans les Documents secrets, l'écrivain belge se demande : " Peut-être pourrait-on dire de mes contes [du recueil Réalités fantastiques] ce que Cocteau écrit à propos de tableaux de Chirico : qu'ils dépaysent la réalité » (Hellens, 1958: 90). Dans le recueil «Six réalités de la guerre », c'est le paysage, cet élément typiquement breughélien, qui est à l'origine du dépaysement.

Le paysage, dans la première édition des Réalités fantastiques, ne constitue pas le fond pour les évènements, mais, il est l'un des acteurs. De cette manière, l'écrivain belge se révèle un disciple fidèle de Breughel qui, sur ses toiles, ennoblit le rôle du paysage en lui attribuant la même importance qu'aux personnages. Nous voudrions souligner que c'est une description poétique de la nature qui se trouve en guise de préface au recueil hellensien : « Je marchais sur le bord d'un étang. C'était une eau sauvage où nulle figure humaine ne s'était encore mirée. [...] Toutes les séductions de la lumière se miraient dans les couleurs. Je rêvais, regardant tour à tour le ciel, les arbres et les buissons fleuris; et tout cela ne faisait qu'un lorsque je ramenais les regards sur l'étang » (Hellens, 1923:1). Ainsi, dès le début, la nature devient "un cadre séduisant» des rêves du narrateur, une invitation au monde d'imagination. Le paysage est aussi le seul témoin de la solitude de l'auteur. Tout au long de "Six réalités de la guerre », nous sentons la solitude du narrateur qui s'écarte de ses compagnons, ce que nous pouvons surtout observer dans le dernier conte, "La méditation devant la mer »: "Les premières hirondelles se croisaient dans l'air blanc et or. Un merle poursuivait dans une ruine sa harangue à petites phrases. Pendant ce temps, le canon faiblissait comme une souffrance qui se guérit à la lumière » (Hellens, 1958: 250). L'observation de la nature devient son refuge qui le protège contre les autres et contre la cruauté de la guerre. 
C'est à travers les descriptions de la nature qu'Hellens introduit un élément du dépaysement, comme dans le passage cité ci-dessous : « On marche sans précaution, à l'aveuglette, les épaules se touchant ; cela fait un clapotement inquiétant mêlé des heurts contre le fer et les pierres. Par moments, ce sinistre morceau de nuit où nous avançons sans nous reconnaître, cette inondation qu'on ne voit pas, mais dont l'approche donne le frisson, ce vent qui harcèle et cette pluie qui frappe le visage, toute cette horreur cachée fait penser à quelque région reculée de l'enfer, accablée d'éternité »(Hellens, 1923 : 230). Le changement de la lumière ou plutôt son manque fait découvrir au narrateur un autre monde dans lequel il ne reconnaît ni soi-même ni ses compagnons. Ainsi la nature provoque une certaine transfiguration de la réalité qui est, en effet, le but d'Hellens. La description de la nature joue donc chez Hellens le rôle du dépaysement qui en fin de compte permet au narrateur de se découvrir soi-même, de se redéfinir. Dans le dernier conte, "Méditation devant la mer », le narrateur, en observant les mouvements des vagues au bord de la mer, intériorise la guerre : "Tout le jeu de la guerre est en toi ; c'est une armée formidable à vaincre » (Hellens, 1923 : 252). Ainsi le paysage ouvre l'accès à l'autre réalité, celle de l'intérieur. Dans le conte hellensien c'est le paysage qui provoque un profond changement intérieur du personnage. Comme le remarque le philosophe français contemporain, Jean-Luc Nancy, «le paysage n'est pas une vue qui “donne sur" quelque perspective [...], c'est plutôt "sur" nous que "donne" une lumière d'inconnu qui vient nous placer en elle et non devant elle » (Nancy, 2003 : 114-115). Dans le cycle « Six réalités de la guerre », c'est, en effet, grâce au paysage, qu'à la fin du cycle, le personnage, après une longue méditation au bord de la mer, retrouve son unité et « comprend la vie sans se l'expliquer» (Hellens, $1923: 253$ ).

Sur l'exemple de «Breughel à l'Yser», il est possible de voir comment l'écrivain passe de «l'identité héritée » à «l'identité reconvertie». Ce conte se révèle comme une étape intermédiaire dans la reconstitution identitaire de Franz Hellens, décrite par Valérie Nahon. L'ouvrage est, d'un côté, un hommage à Pieter Breughel l'Ancien, de l'autre, l'écrivain y construit le monde où règne le principe du fantastique réel. Le fantastique réel, dans cette perspective, semble être issu d'une lecture attentive des toiles du peintre. Hellens réutilise le mythe des écrivains - héritiers des peintres flamands qui a permis d'entamer les premières étapes de la création de l'identité narrative belge et le retravaille d'une manière créative afin de construire sa propre théorie littéraire. Il paraît que l'épanouissement artistique d'Hellens après la guerre ne se déroule pas contre Breughel, mais grâce à lui. Notons que Jean-Marie Klinkenberg voit un lien entre le mythe de l'âme belge et la théorie du fantastique. Selon lui, "l'affirmation critique d'un courant fantastique dans la littérature belge de l'entre-deux guerre peut apparaitre comme une reconversion du mythe nordique de la phase précédente [centripète, J.L.] 》 (Denis, Klinkenberg, 2005 : 188-189). Dans le livre suivant, Mélusine, rédigé en 1917, une année après 
«Six réalités de la guerre », Franz Hellens combine la théorie du fantastique réel avec sa révélation des mouvements avangardistes à portée internationale et il passe ainsi à la phase «cosmopolite» de sa création. Ainsi ce texte pourrait être vu comme un document important dans la relecture de la littérature belge « du temps de doute ». Malheureusement, en rejetant «Six réalités de la guerre », Hellens falsifie une étape importante de sa vie littéraire et aboutit à sa propre déshistoire. 


\section{BIBLIOGRAPHIE}

Baie, E. (1938). « Breughel. Le poète des Gueux » in Le siècle des gueux: histoire de la sensibilité flamande sous la Renaissance (T. III) (pp. 285-448). Bruxelles : Nouvelle Société d'Éditions.

Benjamin, W. (2000). « Sur le concept de l'histoire » in W. Benjamin, Oeuvres III (pp. 427-443), Paris : Gallimard.

Carr, D. (1988). "Epistémologie et ontologie du récit», in J. Greisch, R. Kearney, Paul Riccur. Les Métamorphoses de la raison herméneutique. Colloque de Cerisy-la-Salle (pp. 205-214). Paris : Cerf.

Claessens, B., Rousseau, J. (1969). Notre Bruegel, Anvers : Fonds Mercator.

Denis, B., Klinkenberg, J.-M. (2005). La littérature belge. Précis d'histoire sociale. Bruxelles : Labor.

Frickx, R. (1992). Franz Hellens ou Le Temps dépassé. Bruxelles : Palais des Académies.

Hellens, F. (1923). Réalités fantastiques. Bruxelles : Disque vert.

- (1958). Documents secrets : histoire sentimentale de mes livres et de quelques amitiés. Paris : Éditions Albin Michel.

- (1967). Le Fantastique réel. Amiens : Éditons Sodi.

- (1992). Un balcon sur l'Europe. Bruxelles : Labor.

Nahon, V. (2009). «Les Documents secrets ou la construction d'une image de soi : Franz Hellens et l'identité de l'écrivain-peintre », Textyles, 35, 115-130, http://textyles.revues.org/188.

Nancy, J.-L.(2003). «Paysage avec dépaysement » in Au fond des images (pp. 101-120). Paris : Galilée.

Quaghebeur, M. (1998). Balises pour l'histoire des lettres belges. Bruxelles : Labor.

Van Mander, C. (1884). Le Livre des peintres. Vie des peintres flamands, hollandais et allemands, Paris : Librairie de l'art., consulté sur le site gallica fr.

Van Puyvelde, L. (1962). La peinture flamande au siècle de Breughel et de Bosch. Paris : Elsevier. 
